\title{
Non-Linear Relationship between Heart Period and Root Mean Square of Successive Differences during Ramp Exercise and Early Recovery
}

\author{
MJ Gaitán-González ${ }^{1}$, S Carrasco-Sosa ${ }^{1}$, R González-Camarena ${ }^{1}$, O Yáñez-Suárez² \\ ${ }^{1}$ Depto. Ciencias de la Salud, Universidad Autónoma Metropolitana-Iztapalapa, Mexico City, Mexico \\ ${ }^{2}$ Depto. Ingeniería Eléctrica, Universidad Autónoma Metropolitana-Iztapalapa, Mexico City, Mexico
}

\begin{abstract}
To compare the relationships between heart period and root mean square of successive differences for exercise and recovery, these parameters were obtained in eight subjects during three one-minute successive submaximal workloads of cycle exercise and the first three minutes of recovery, using a 25 second sliding window. In most subjects, at recovery, the variables reached values over those at resting condition. Individual semi-log and log-log regressions were estimated and the regression parameters for both stages were compared by paired $t$ test. All correlations were very strong, higher for the exercise stage, and the regressions were slightly better for the log-log case. The parameters had significant differences between exercise and recovery stages, showing that the relationship between the heart period and the vagal modulation index changed with the subject condition, in this case due to the differences in the response of both variables during the recovery stage.
\end{abstract}

\section{Introduction}

Root mean square of successive differences (rMSSD) is a time-domain heart rate variability index that has been related to the heart vagal modulation and which is easy to compute [1]. A significant non-linear relationship has been observed between heart period [2] or heart rate [3] and this index. Using a wide range of cardiac frequencies, the relationship is so strong [2] that it would be questionable if those measures are surrogated. If that were the case, the relationship should remain for different physiological conditions. However, studies where the correlation has been obtained [2-4] do not test it for different physiological states under the same heart period range, and the data used for computing the regressions were obtained from different individuals. Intra-subject relationships have not been assessed.

To obtain the intra-subject vagal modulation-heart tone relationship (rMSSD vs. mean heart period), the use of maneuvers that ensure enough data samples and heart period range of evaluation would be appropriate. Dynamic ramp exercise is an experimental condition where, as a function of the workload, the heart rate increases, diminishing the heart period, with a vagal modulation withdraw [5]. This condition might present a range of cardiac period which is wide enough to assess the vagal modulation - tone relationship, however, the usual assumption of having a steady state condition for several minutes, is not met during ramp exercise.

For the post-exercise recovery this limitation is also present and may be even stronger, since the variable properties are changing during this transitory state. The heart rate variability behavior for the first minutes of post-exercise recovery has not been fully assessed, and the evaluation of "early recovery" is done after the transitory stage [6,7]. Exercise recovery is a state where the ranges of heart period and vagal modulation index are expected to be similar to those obtained for the exercise condition, with a progression occurring in the opposite direction, that is, an increase in both heart period and vagal modulation.

The goal of the present work was to compare the nonlinear regressions between rMSSD and mean heart period during both dynamic sub-maximal ramp exercise and early post-exercise recovery, using modified, timevarying indexes.

\section{Methods}

Eight subjects, three women and five men, life-long residents of Mexico City participated in the study. Their anthropometric measurements, expressed as mean \pm standard deviation, were: height, $164.1 \pm 8.4 \mathrm{~cm}$ and weight, $61.0 \pm 10.6 \mathrm{Kg}$. Subjects were healthy as established by clinical examination, electrocardiogram at rest, and spirometry; young ( $23.0 \pm 1.7$ years old); nonsmokers, and sedentary. Their written informed consent was requested to participate. None of the subjects took any food, alcoholic or stimulant beverages, nor performed intense physical activity 12 hours before the study.

Exercise was performed using a bicycle ergometer (818E, Monark, Vargberg, Sweden). Exercise stage 
consisted on successive one minute workloads of 25,50 and 75 watts, followed by a three minutes recovery stage in sitting position (the subject remained seated on the ergometer). One pre-exercise minute with the subject in sitting position was obtained to define initial conditions.

The electrocardiogram was detected through three floating electrodes, by means of bipolar lead CM5, using a monitor (78330A Hewlett Packard, USA). Electrocardiogram was acquired at $500 \mathrm{~Hz}$ of sampling frequency. None of the participants presented ectopic beats.

Maximum values for $\mathrm{R}$ waves were detected to generate temporal series of the RR intervals. Mean heart period (mean RR) and rMSSD were computed for each stage, using a sliding window of $25 \mathrm{~s}$.

Individual semi-log and log-log regressions between the rMSSD and the heart period were estimated for the exercise and recovery stages, and paired t-tests were performed to compare each of the regression parameters of both conditions. Statistical significance was accepted at $\mathrm{p}<0.05$.

\section{Results}

At rest, the mean heart period was $767 \pm 96 \mathrm{~ms}$, while the rMSSD was $38.1 \pm 23.5 \mathrm{~ms}$. During exercise, the mean heart period and vagal modulation index decreased accordingly with the ramp exercise load. Around the end of the exercise, the heart period reached its minimum, which was of $515 \pm 55 \mathrm{~ms}$. For recovery stage, the mean heart period tendency was to return gradually to its initial

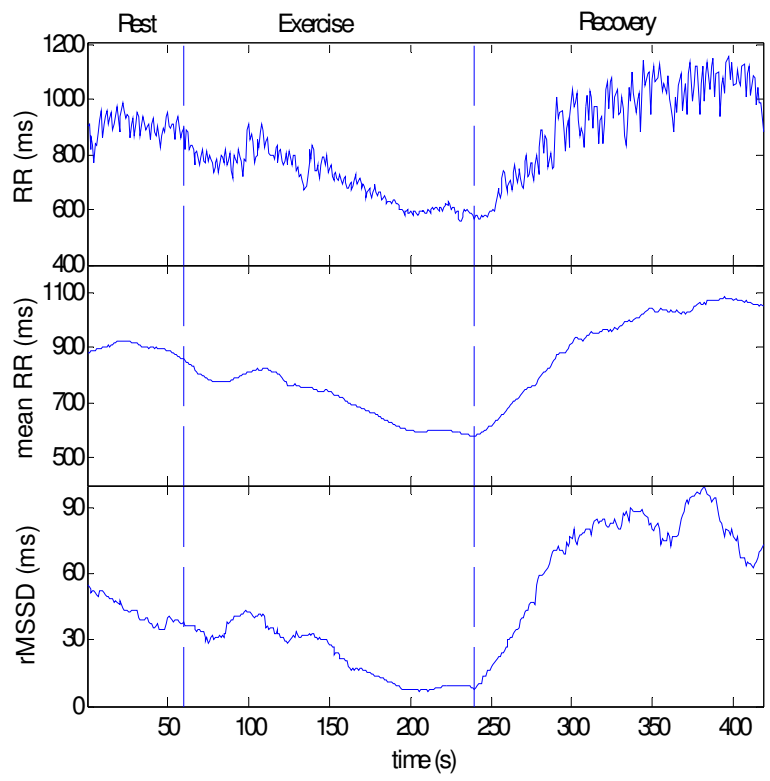

Figure 1. RR interval, mean heart period (mean RR), and rMSSD. The last two variables were obtained using a $25 \mathrm{~s}$ sliding window. value while the vagal index increased sharply after the exercise finished. In most subjects, both the vagal modulation index and the heart period reached values over those at rest condition, tending to later return to values near the initial level. Figure 1 presents an example of the temporal behavior of the RR intervals, the mean heart period and the rMSSD, during the previous minute to the test, with the individual sitting at rest, and the exercise and recovery stages.
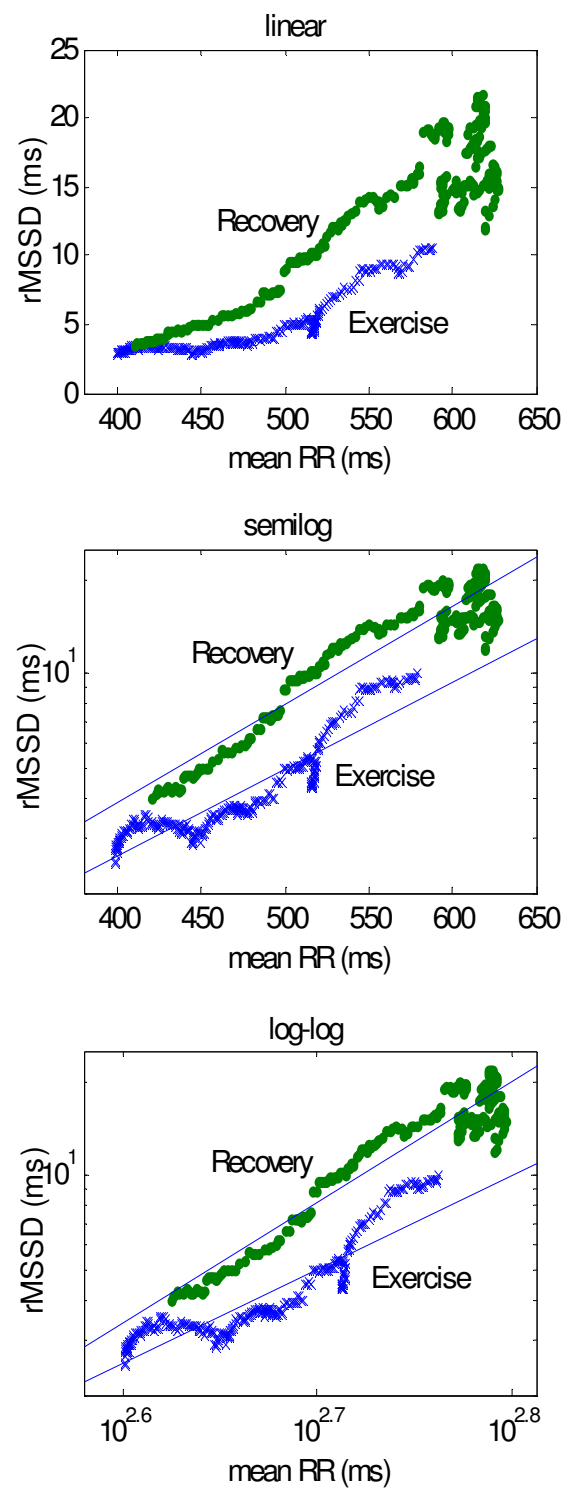

Figure 2. Example of linear, semi-log and log-log scatter plots of mean heart period (mean RR) and rMSSD obtained during ramp exercise (.) and post-exercise recovery $(\mathrm{x})$ using a $25 \mathrm{~s}$ sliding window. Estimated regression lines are plotted over the respective data. 
Table I. rMSSD vs. mean RR log-log and semi-log regression parameters

\begin{tabular}{ccccc}
\hline & $\mathrm{r}$ & Slope & Intercept & Residual variance \\
\hline $\log -\log$ & & & & \\
Exercise & $0.96 \pm 0.04$ & $4.87 \pm 0.84$ & $-28.8 \pm 5.5$ & $0.01 \pm 0.00$ \\
& $(0.88,0.99)$ & $(3.85,6.10)$ & $(-36.8,-20.8)$ & $(0.01,0.02)$ \\
Recovery & $0.90 \pm 0.07 *$ & $3.45 \pm 0.76 *$ & $-19.1 \pm 4.9 *$ & $0.05 \pm 0.04 *$ \\
& $(0.78,0.9)$ & $(2.37,4.29)$ & $(-24.5,-11.6)$ & $(0.01,0.13)$ \\
semi-log & & & & \\
Exercise & $0.95 \pm 0.03$ & $0.008 \pm 0.001$ & $-2.4 \pm 0.5$ & $0.01 \pm 0.01$ \\
& $(0.88,0.99)$ & $(0.006,0.009)$ & $(-3.1,1.7)$ & $(0.01,0.02)$ \\
Recovery & $0.89 \pm 0.07 *$ & $0.005 \pm 0.002^{*}$ & $-0.1 \pm 1.2 *$ & $0.07 \pm 0.05 *$ \\
& $(0.73,0.95)$ & $(0.003,0.008)$ & $(-1.9,1.9)$ & $(0.01,0.14)$ \\
\hline
\end{tabular}

Mean \pm standard deviation, range of values in parenthesis.

* Significant differences with Exercise, $\mathrm{p}<0.05$

A typical scatter plot of the rMSSD and the mean heart period, as well as the log-log and semi-log relationships are shown in Figure 2. In general, for the first couple of minutes of recovery, the vagal modulation index showed higher values for the same heart period, than those observed for the exercise stage. At the end of the recording, the rMSSD-mean heart rate relationship tended to change its behavior pattern.

Table 1 presents the $\log -\log$ and semi-log regression parameters. Strong log-log correlations between heart period and vagal heart rate variability indexes were observed, higher for the exercise stage than for the recovery stage. The individual correlation coefficients for the semi-log case were slightly lower, and the residual variance larger.

All the regression parameters, the correlation coefficient, the slope, the intercept, and the residuals variance, showed significant differences between the exercise and recovery stages $(p<0.05)$ for both types of regressions.

\section{Discussion and conclusions}

The main findings of this research were: (a) the maneuvers and the modified indexes (averaged using a $25 \mathrm{~s}$ sliding window) allowed the assessment of the heart period and rMSSD relationship in an intra-subject basis, (b) the vagal modulation behavior during the first three minutes of recovery presented an overshoot, (c) there were very strong intra-subject non-linear relationships between both variables, (d) although these relationships were very strong, they showed significant differences with the maneuver for the same heart period range.

Exercise and recovery resulted in a couple of maneuvers where the heart rate and autonomic system response varied enough to assess the relationship among the mean heart period and the vagal modulation index in an individual basis, the intra-subject relationship. Besides, the use of a sliding window allowed the assessment of the rMSSD and the mean heart period in a non-stationary maneuver.

The minimum data length for heart rate variability indexes estimation has been explored by other authors $[8,9]$. They concluded that the vagal indexes, in particular the rMSSD, have less dependency over the data length. Hamilton et al. [8] did not found significant differences for its assessment in a 10s window when compared with the computation on a five-minute interval, while McNames et al. [9] evaluated segments of different length and compared them with the respective five-minute estimation. The comparison made by these authors include other measures beside bias to evaluate the reliability of using shorter or longer than five minutes data segments, a traditional length for short-term heart rate variability assessment. In the present work, the selected sliding window length was short enough to observe the evolution of the heart rate variability index within an acceptable reliability.

The RR interval decreased with the workload increments. During the exercise there is a vagal withdraw and a sympathetic promotion. This can be observed in the reduction of the heart period together with the decrement on the global heart rate variability (top of Figure 1); in particular, there was a reduction in the parasympathetic modulation, observable in the decrease of the rMSSD (bottom of Figure 1).

During the first minute of recovery, the RR interval series (top of Figure 1) showed an increment on the respiratory sinus arrhythmia amplitude; the vagal modulation restoration was fast, as observed through the rMSSD, and remained altered after the recovery period under observation (bottom of Figure 1). Even more, in 
most subjects, both signals, but specially the vagal modulation index, reached values over those at rest condition.

Semi-log and log-log relationships have been used to relate inter-subject mean heart period and rMSSD [2-4]. In the present work, both regressions presented high correlation values for intra-subject assessment, although log-log regression was slightly better for most of the participants. Correlation coefficients larger than those previously reported [2-4] were found, probably due to a larger domain of assessment.

This corroborate that there is a strong correlation between these two variables for both intra- and intersubject data. However, since all the regression parameters presented significant differences for both conditions, we consider that the relationship between mean heart rate and heart vagal modulation changed with the subject physiological condition. Whether this behavior is also present for other physiological stages should be studied as well as the mechanisms involved in this difference on performance.

We conclude that there were very strong non-linear intra-subject relationships between the mean heart period and the rMSSD for exercise and recovery, but these relationships changed with the physiological condition. The fast increase of the vagal modulation index once the exercise was finished, together with a gradual increase in the heart rate and the overshoot observed in post-exercise recovery, might produce such behavior.

\section{References}

[1] Task Force of the European Society of Cardiology and the North American Society of Pacing and Electrophysiology. Heart rate variability: standards of measurement, physiological interpretation, and clinical use. Circulation 1996; 93:1043-65.
[2] Carrasco S, Gaitán MJ, González R, Yáñez O. Correlation among Poincaré plot indexes and time and frequency domain measures of heart rate variability. J Med Eng Technol 2001; 240-8.

[3] Ziegler D, Strabbuger K, Lambech H, Dannelh K. Normal ranges and reproducibility of statistical, geometric, frequency domain, and non-linear measures of 24-hour heart rate variability. Horm Metab Res 1999; 31: 672-9.

[4] Person A, Solders G. R-R variations, a test of autonomic disfunction. Clin Sci 1982; 61:379-83.

[5] Tulpo LP, Mäkikallo TH, Seppänen T, Laukkanen RT, Huikuri HV. Vagal modulation of heart rate during exercise: effects of age and physical fitness. Am J Physiol 1998; 274: H424-9.

[6] Carrasco S, González R, Gaitán M, .Yáñez O Reproducibility of heart rate variability for short-term recordings during five maneuvers for normal subjects. J Med Eng Technol 2003; 27:241-8.

[7] Du N, Bai S, Oguri K, Kato Y, Matsumoto I, Kawase H, Matsuoka T. Hear rate recovery after exercise and neural regulation of heart rate variability in $30-40$ year female marathon runners. J Sport Sci Med 2005; 4:9-17.

[8] Hamilton RM, Mckechnie PS, Macfarlane PW. Can cardiac vagal tone be estimated from the 10-second ECG? Int J Cardiol 2004; 95:109-15

[9] McNames J, Thong T, Goldstain B. Reliability and accuracy of heart rate variability metrics versus ECG segment duration. 25th Annual International Conference Engineering in Medicine and Biology Society, Cancun, 2003: $212-5$.

Address for correspondence

Mercedes Jatziri Gaitán González

Lab. Fisiología Humana. Depto. Ciencias de la Salud. Universidad Autónoma Metropolitana Iztapalapa.

Av, San Rafael Atlixco 186 Col. Vicentinas

09340, México City, México

e-mail:mjgg@xanum.uam.mx 